\title{
Aislamiento de Erysipelothrix rhusiopathiae asociado a endocarditis en cerdos de Guadalajara, J alisco
}

\author{
Isolation of Erysipelothrix rhusiopathiae asociated with \\ endocarditis in pigs in Guadalajara, J alisco
}

\author{
María de Jesús De Haro-Cruz ${ }^{\text {** }}$, Sonia Gutiérrez-Paredesa , Christian Zavala-Escobara , Fernando Martín \\ Guerra-Infante ${ }^{a, b}$, Emilio Campos-Morales ${ }^{c}$
}

\begin{abstract}
RESUMEN
Erysipelothrix rhusiopathiae es el agente causal de la erisipela porcina, enfermedad que ocasiona pérdidas económicas importantes en las explotaciones porcinas. En México se desconoce la presencia de la infección, principalmente porque el cuadro clínico se presenta acompañado por otros patógenos, además el aislamiento es difícil por los requerimientos de la bacteria. En un rancho porcícola de Guadalajara se dieron a conocer casos de endocarditis, disminución en la producción y observación de manchas rojizas en la piel de animales jóvenes y las hembras de pie de cría, por lo que se buscó la presencia del microorganismo en la explotación. Para realizar el aislamiento de la bacteria se recolectaron 8 muestras de sangre, 11 de corazón, 6 de amígdalas y 3 de líquido sinovial de cerdos de siete meses de edad y de hembras de pie de cría. Las muestras se sembraron en caldo selectivo para Erysipelothrix, los órganos se sembraron en gelosa sangre, medio de Packer modificado y agar soya tripticaseina suplementado con suero de caballo. A partir de líquido sinovial, corazón y amígdalas se logró el aislamiento de cinco cepas identificadas como $E$. rhusiopathiae, lo que fue confirmado por medio del sistema API-Coryne. Estos hallazgos determinan la presencia de $\boldsymbol{E}$. rhusiopathiae como causante de endocarditis en cerdos de Guadalajara, Jalisco, así como la necesidad de determinar su presencia en granjas porcícolas del país para conocer su importancia epidemiológica.
\end{abstract}

PALABRAS CLAVE: Erysipelothrix rhusiopathiae, Artritis, Endocarditis, Septicemia, Cerdos.

\section{ABSTRACT}

Erysipelothrix rhusiopathiae is the causative agent of swine erysipelas, a disease that causes significant economic losses in pig farms. In Mexico, the presence of this infection is unknown, mainly because the clinical form is accompanied by other pathogens. Furthermore, the isolation of the species is difficult due to the requirements of the bacteria. On a hog ranch in Guadalajara, cases of endocarditis, decreased production and observations of red spots on the skin of young animals and female broodstock were observed. Thus, the causative organism of these symptoms was sought. For the isolation of the bacterium were collected 8 samples of blood, 11 samples of heart, 6 samples of tonsils and 3 samples of synovial fluid of pigs of 7 mo of age and females' broodstock. Blood samples were seeded in broth selective for Erysipelothrix. Organs were plated on blood agar and modified tryptone soy agar supplemented with horse serum. Five $E$. rhusiopathiae strains were isolated from the synovial fluid, hearts and tonsils and these were confirmed using API-Coryne system. These findings confirmed that $E$. rhusiopathiae caused endocarditis in swine in Guadalajara, Jalisco, Mexico and the necessity to determine its presence in pig farms in the country to know their epidemiological importance.

KEY WORDS: Erysipelothrix rhusiopathiae, Endocarditis, Arthritis, Septicemia, Pigs.

Recibido el 5 de abril de 2016. Aceptado el 9 de septiembre de 2016.

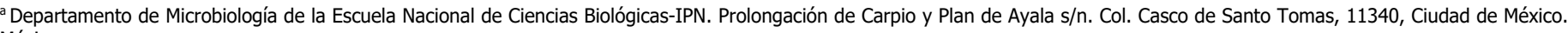
México.

b Laboratorio de Virología del Instituto Nacional de Perinatología. México.

${ }^{c}$ Centro Universitario de Ciencias Biológicas y Agropecuarias-UDG. México

*Autor de correspondencia: deharoc@yahoo.com.mx. 
Erysipelothrix rhusiopathiae es el agente causal de erisipela porcina, de septicemia en aves, además de erisipeloide en humanos ${ }^{(1,2)}$. La erisipela de los cerdos se ha aislado en Europa, Asia, África, América, Japón, China, Estados Unidos, Australia, Brasil y Chile entre otros. La enfermedad causada por este microorganismo causa serias pérdidas económicas en la industria ganadera debido a la muerte de los cerdos y a la devaluación de las canales por la artritis $^{(3-5)}$.

Los signos clínicos en cerdos pueden ser agudos, subagudos y crónicos. La forma subaguda se caracteriza por lesiones en la piel con forma de diamante que progresan a una forma aguda ocasionando infección septicémica y muerte. La forma crónica sigue de la infección aguda, causando daño en las válvulas cardiacas (endocarditis) y articulaciones (artritis) ${ }^{(5,6)}$.

Erysipelothrix rhusiopathiae se aísla principalmente de tonsilas 0 de lesiones de endocarditis y artritis a partir de cuadros clínicos agudos o crónicos. La prevalencia de la infección en cerdos portadores oscila entre 3 y $98 \%$; sin embargo debido al aumento de las explotaciones cerradas para el ganado porcino, y la ausencia de contacto con terreno contaminado, la incidencia de la enfermedad ha disminuido notablemente ${ }^{(5,7)}$.

En México la presencia del microorganismo se desconoce, ya que actualmente no existen estudios epidemiológicos que determinen su incidencia en el país, debido principalmente a que se encuentra asociado a otros patógenos y su aislamiento es difícil. Es por esto que se deben realizar más estudios para determinar la prevalencia de la enfermedad y si puede estar afectando la economía mexicana.

En una granja de ciclo completo ubicada en la población de la Cofradía, Tlajomulco de Zúñiga, Jalisco, municipio que conforma parte de la zona conurbada de Guadalajara, se manifestaron problemas en el área de gestación con la aparición de abortos en cerdas con 50 a 65 días de gestación, en algunas de ellas con la aparición de orejas inflamadas; estos animales recibieron tratamiento a base de penicilina, mostrando recuperación; sin embargo las orejas no se recuperaron quedando arrugadas como de cartón corrugado (oreja de chicharrón o acartonada). En el área de destete se apreciaron lesiones en la punta y en la parte inferior de las orejas de ellas; otros lechones presentaban un andar doloroso e inflamación de las articulaciones del carpo. En el área de engorda se observaron animales con fiebre, anorexia, cianosis en las región ventral del cerdo, en dos casos se observaron manchas rojas en la piel simulando un rombo. La cantidad de animales enfermos clínicamente fueron alrededor de $8 \%$, sin embargo aproximadamente el $40 \%$ de la granja presentaba al menos uno de los signos de la enfermedad.

De acuerdo a estos datos clínicos se sospechó de la presencia de E. rhusiopathiae, por lo que se realizó la necropsia de tres cerdos muertos del área de engorda, se inspeccionaron los pulmones no encontrando cambios significativos; en el corazón se encontraron las válvulas engrosadas, pareciendo una endocarditis vegetativa, esplenomegalia, inflamación de las articulaciones del carpo y metacarpo y el líquido sinovial de color ámbar (Figura 1). A partir de estos animales muertos por

Figura 1. Cerdos con signos de erisipela porcina. a) daño a nivel de orejas (0); b) cerdo muerto de septicemia; c) manchas rojizas en la piel (R) caracteristicas de erisipela; d) y e) lesiones de endocarditis, f) presencia de abortos en el día 65 de gestacion.

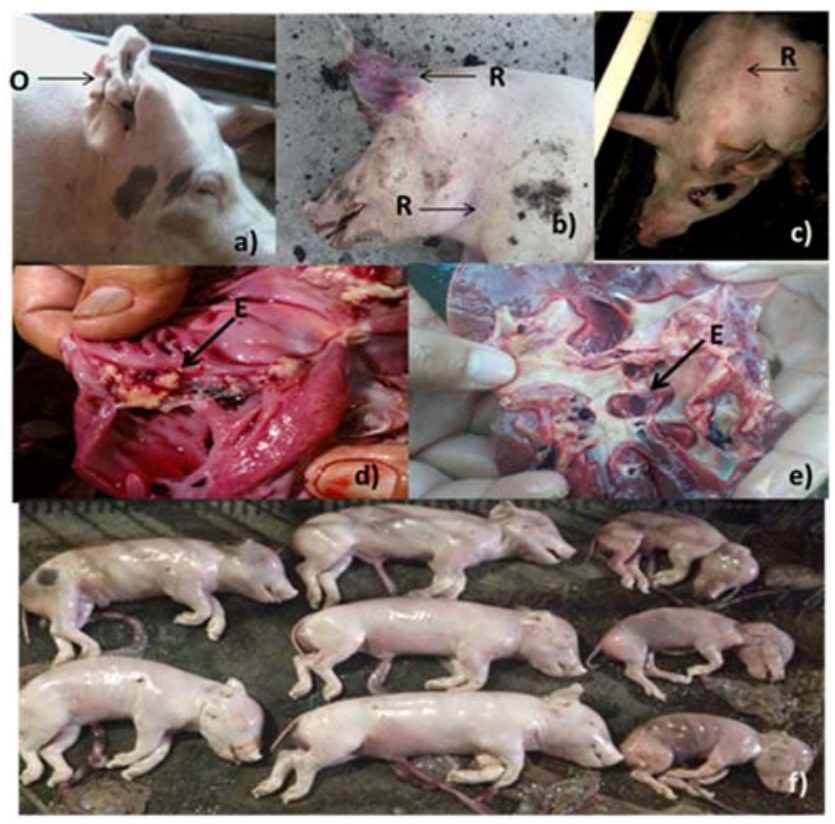


septicemia, de animales en el área de destete y de las cerdas del área de gestación, se tomaron y analizaron 28 muestras, de las cuales 3 fueron de líquido sinovial, 8 de sangre, 11 de corazón y 6 de amígdalas.

Las muestras de tejido se colocaron en frascos con medio de transporte de Stuart, en tanto que las muestras de sangre se obtuvieron en tubos con heparina para evitar su coagulación. Las muestras de órgano se maceraron y se sembraron en agar sangre, agar soya tripticaseína complementado con $3 \%$ de suero de caballo y medio de Packer modificado. Los medios de cultivo se incubaron a 37 ${ }^{\circ} \mathrm{C}$ durante 24 a 48 h. Las colonias con una morfología microscópica y colonial compatible a Erysipelothrix sp fueron resembradas en agar soya tripticaseína con 3\% de suero de caballo y agar sangre para su posterior identificación fenotípica mediante el sistema API Coryne System ${ }^{(4)}$, de acuerdo a las instrucciones del fabricante, utilizando adicionalmente pruebas de movilidad, producción de ácido sulfhídrico y reacción negativa a la catalasa y oxidasa. El diagnóstico diferencial de realizó mediante la búsqueda de Mycoplasma hyorinis, Haemophylus parasuis, y artritis por estafilococos o estreptococos.

A partir de las muestras de líquido sinovial, amígdalas y corazón, se aislaron ocho cepas sospechosas de Erysipelothrix sp, de las cuales cinco fueron identificadas como $E$. rhusiopathiae y tres como $E$. tonsillarum de acuerdo a su perfil bioquímico. E. tonsillarum se aisló a partir de las muestras de amígdalas. No se logró el aislamiento de ningún microorganismo a partir de las muestras de sangre.

La bacteria que ocasiona el cuadro clínico de erisipela, en apariencia es una bacteria sin importancia en la clínica de porcinos, ya que por su patogenicidad pasa desapercibida en la mayoría de los casos; sin embargo es de importancia económica, ya que su efecto patológico causa retraso en el desarrollo de los cerdos, problemas reproductivos en sementales como orquitis, y ocasionando necrosis de los tejidos que conforman el testículo; en cerdas reproductoras causa lesiones y necrosis del ovario, además de ser una bacteria que afecta a la salud pública, ya que puede infectar a los trabajadores. Las pérdidas económicas son cuantiosas, ya que el retraso en la engorda del cerdo es de aproximadamente 28 días $^{(8,9)}$.

En la granja porcina de La Cofradía las cerdas reproductoras son inmunizadas con la aplicación de la vacuna muerta combinada para la prevención de parvovirus porcino, leptospirosis y erisipela, aplicándose a las cerdas adultas 15 días después del parto; los reemplazos son vacunados al momento de integrarlos al plantel de producción, repitiéndose el esquema 15 días después.

Los datos clínicos característicos de infección por $E$. rhusiopathiae se observaron en los cerdos de destete y de engorda, lo que significa que la vacuna utilizada en la granja mantiene una corta protección; esto indica que existen animales portadores encargados de diseminar a la bacteria, ocasionando el retraso en el crecimiento e incluso la muerte de los animales con los cuadros clínicos que se mencionaron.

El diagnóstico de la enfermedad se realiza principalmente con el aislamiento en medios selectivos, y con la identificación del microorganismo mediante pruebas bioquímicas convencionales ${ }^{(10)}$. En este estudio se obtuvieron ocho aislamientos a partir de muestras de corazón, líquido sinovial y amígdalas, de los cuales cinco fueron identificados como E. rhusiopathiae utilizando el sistema APY Coryne System, siendo los tres aislamientos restantes identificados como $E$. tonsillarum.

En este estudio no se encontró la presencia de algún otro microorganismo causante de artritis o septicemia, por lo que los problemas de artritis, endocarditis, septicemia y abortos de la granja muestreada son debidos a $E$. rhusiopathiae.

El aislamiento y la identificación de $E$. rhusiopathiae se considera difícil, ya que es una bacteria que requiere medios de cultivo especiales suplementados con suero, sangre o antibióticos que impidan el crecimiento de biota acompañante; el crecimiento es lento, además de que se debe hacer diferenciación entre $E$. rhusiopathiae y $E$. tonsillarum, principalmente en muestras de amígdalas, por lo que existen pocos estudios en México en donde se realice su aislamiento $e$ identificación ${ }^{(3,10,11)}$. 
Los resultados de este trabajo demuestran la presencia de E. rhusiopathiae en una granja de la región conurbada de Guadalajara, encontrándose problemas de endocarditis, así como retrasos en el crecimiento y pérdidas económicas a pesar de que se utiliza una vacuna contra la erisipela. Esto podría estar indicando que las vacunas comerciales no están protegiendo de manera efectiva contra los serotipos prevalentes en la zona. Más estudios se requieren para analizar la presencia de $E$. rhusiopathiae en las granjas porcícolas de la entidad para determinar la prevalencia del microorganismo en la zona, y así definir la conveniencia de utilizar otro tipo de vacuna que logre una mejor protección.

\section{LITERATURA CITADA}

1. Harada K, Uchiyama M, Hoshi T, Takahashi T. Comparison of three DNA extraction methods for detection of Erysipelothrix rhusiopathiae in chicken blood by polymerase chain reaction. J Vet Diagnost Invest 2009;(21):354-358.

2. Ingebritsona AL, Rothb JA, Hauerc PJ. Erysipelothrix rhusiopathiae: Association of Spa-type with serotype and role in protective immunity. Vaccine 2010;(20):2490-2496.

3. Shimoji $Y$, Yokomizo $Y$, Mori $Y$. Intracellular survival and replication of Erysipelothrix rhusiopathiae within murine macrophages: Failure of induction of the oxidative burst of macrophages. Infect Immun 1996;64(5):1789-1793.

4. Soto A, Zapardiel J, Soriano F. Evaluation of API Coryne system for identifying coryneform bacteria. J Clin Pathol 1994;(47):756-759.

5. Stackebrandt E, Reboli AC, Farrar WE. The genus Erysipelothrix. Prokaryotes 2006;(4):492-510.

6. Takahashi T, Sunama P, Satra J, Cholsindhu N, Kongthon S, Jitnupong W, et al. Serotyping and pathogenicity of Erysipelothrix strains isolated from tonsils of slaughter pigs in Thailand. J Vet Med Sci 1999;61(9):1007-1011.

7. Takeshi K, Makino S, Ikeda T, Takada N, Nakashiro A, Nakanishi K, et al. Direct and rapid detection by PCR of Erysipelothrix sp. DNAs prepared from bacterial strains and animal tissues. J Clin Microbiol 1999;37(12):4093-4098.

8. To $\mathrm{H}$, Sato $\mathrm{H}$, Tazumi A, Tsutsumi N, Nagai S, Iwata A, et al. Characterization of Erysipelothrix rhusiopathiae strains isolated from recent swine erisipelas outbreaks in Japan. J Vet Med Sci 2012;(74):949-953.

9. Restrepo-Salazar JG, Agudelo-López SP, Vélez-Aramburu E, Orrego-Cardona JC. Determinación de la seroprevalencia de Erisipela en cerdos de Lomarena - Bolívar mediante ELISA. CES Med Vet Zootec 2008;3(1):17-26.

10. To $\mathrm{H}$, Nagai S. Genetic and antigenic diversity of the surface protective antigen proteins of Erysipelothrix rhusiopathiae. Clin Vacc Immunol 2007;(14):813-820.

11. Yamazaki Y. A multiplex polymerase chain reaction for discriminating from Erysipelothrix tonsillarum. J Vet Diagn Invest 2006;(18):384-387. 